\title{
Diagnostic technique for Zeeman-compensated atomic beam slowing: Technique and results
}

\author{
P. A. Molenaar, P. van der Straten, and H. G. M. Heideman \\ Debye Institute, Department of Atomic and Interface Physics, Utrecht University, P.O. Box 80.000, 3508 TA Utrecht, The Netherlands \\ H. Metcalf \\ Physics Department, State University of New York at Stony Brook, Stony Brook, New York 11794
}

(Received 25 March 1996; revised manuscript received 26 June 1996)

\begin{abstract}
We have developed a diagnostic tool for the study of Zeeman-compensated slowing of an alkali-metal atomic beam. Our time-of-flight technique measures the longitudinal velocity distribution of the slowed atoms with a resolution below the Doppler limit. Furthermore, it can map the position and velocity distribution of atoms in either ground hyperfine level inside the solenoid without any devices inside the solenoid. The technique reveals the optical pumping effects and shows in detail how the slowing within the solenoid proceeds. We find for $\mathrm{Na}$ that most atoms in the chosen hyperfine state are decelerated in the slowing process. The width of the velocity distribution is determined mainly by inhomogeneities in the slowing laser beam. Using the central most uniform part of an expanded laser beam, the width is reduced to $2.5 \mathrm{~m} / \mathrm{s}$, corresponding to 3.2 $\mathrm{mK}$. Finally, we discuss and show a method to produce a beam with two-velocity components for the study of head-tail low-energy collisions. [S1050-2947(97)03101-6]
\end{abstract}

PACS number(s): $32.80 . P \mathrm{j}, 42.50 . \mathrm{Vk}$

\section{INTRODUCTION}

Deceleration of atoms in a thermal beam using a single beam of counterpropagating laser light has been achieved in many laboratories. In many cases the slowed atoms are used to load a magneto-optical trap (MOT), for which the capture velocity, typically $30 \mathrm{~m} / \mathrm{s}$, is well below the thermal velocity of atoms. Then the only requirement for the slowing process is that it produce enough atoms with a velocity below the capture velocity. Furthermore, since the density in a MOT is limited by collisions between trapped atoms, increasing the flux of slowed atoms in general does not increase the density in the MOT.

In the present paper we explore the optimization of such atom deceleration for the production of a slow and cold atomic beam for collision experiments. This puts extra requirements on the preparation of the atomic beam. First, the atoms in the beam should be sufficiently monovelocity, i.e., the velocity spread of the atoms should be considerably smaller than the average velocity (cold). Second, since any collision signal is directly proportional to the flux of atoms, it is important to achieve a flux as high as possible. Our study concentrates on these aspects of the slowing process.

The most common way to measure the slowed velocity distribution depends on detecting the fluorescence from atoms excited by a second laser beam propagating at a small angle to the atomic beam. Because of the Doppler shift, the frequency dependence of this fluorescence provides a measure of the atomic velocity distribution. In this paper we describe a time-of-flight (TOF) method to accomplish the same result, however, with a much improved resolution. In addition it provides a much more powerful diagnostic of the deceleration process.

Beam slowing requires compensation of the changing Doppler shift to maintain resonance between the decelerating atoms and the laser beam used for slowing. This has been accomplished in many different ways $[1-5]$, but the first and most widely used method is the Zeeman technique [5-8]. One of the main advantages of the Zeeman technique over others is the ability to slow all atoms from the source into a continuous beam, and this makes the technique most suitable for collision experiments. It uses an inhomogeneous magnetic field along the deceleration path to tune the resonance frequency of the moving atoms spatially and thereby maintain the deceleration process. The TOF method we present here for sodium has the capability to map out the velocity distribution for both hyperfine ground states. Furthermore, this mapping of the velocity distribution can be achieved along the entire path of the atoms throughout the solenoid. Pumping of atoms in the wrong hyperfine state or losing atoms during the slowing process therefore can easily be detected and in most cases corrected.

\section{EXPERIMENTAL SETUP}

The experimental arrangement is shown in Fig. 1. The atoms emerge through an aperture of $1 \mathrm{~mm}^{2}$ from an effusive sodium source heated to approximately $300^{\circ} \mathrm{C}$. They

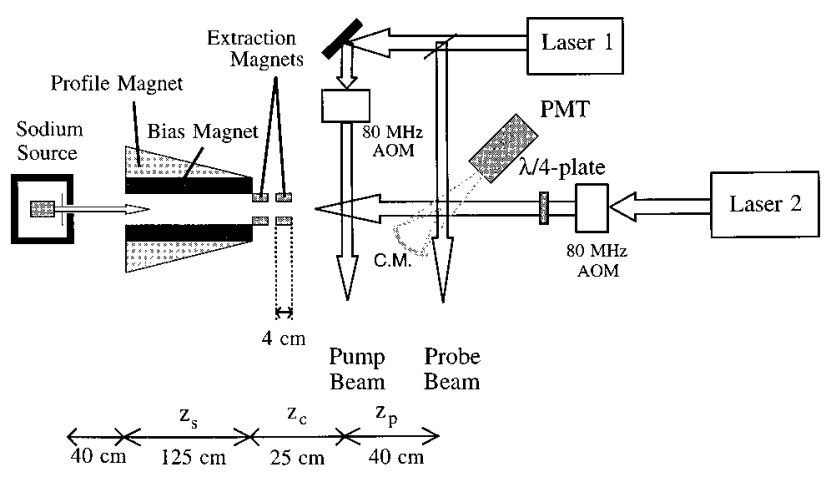

FIG. 1. Diagram of the apparatus showing the positions of the magnets and laser beams. 


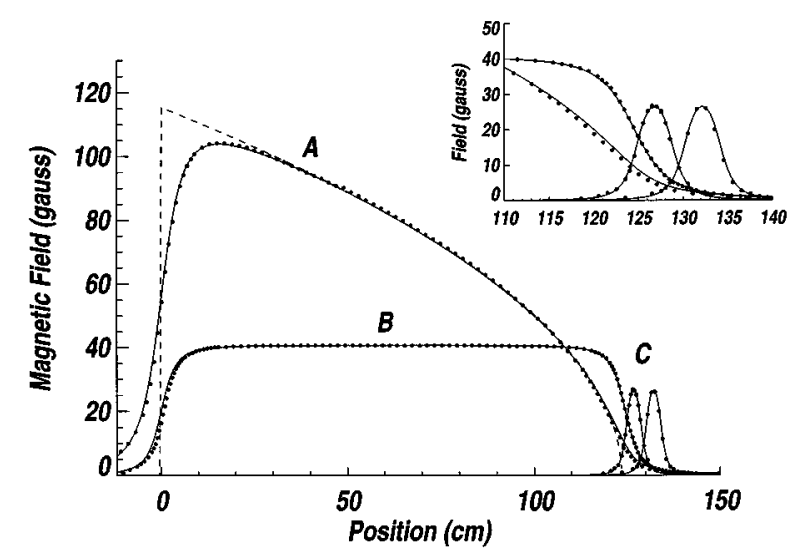

FIG. 2. Plot of the measured spatial dependence of the magnetic fields at a current of $1 \mathrm{~A}$ through all windings (dots). The labels $A, B$, and $C$ indicate the profile, bias, and extraction coils, respectively. The solid lines represent the field designs. The dashed line shows the idealized square-root form of the profile magnetic field. The inset shows the magnetic field near the extraction region for the different solenoids.

then travel $40 \mathrm{~cm}$ to the solenoid. During the flight through the solenoid the atoms are slowed by the counterpropagating laser light. To minimize the effect of transverse spreading of the atoms during the slowing process, we focus the laser beam on the aperture of the oven. The diameter of the slowing laser at the point where the atoms leave the solenoid is $\approx 9 \mathrm{~mm}$. To keep the vacuum low during the slowing process we honed the interior walls of the solenoid.

There are three different magnet windings along the solenoid core, all wrapped with $3 \times 1 \mathrm{~mm}^{2}$ rectangular copper wire. The first one consists of ten layers along the whole length of the core and it produces a homogeneous bias magnetic field $B_{b}$. The tapered field of the second winding produces the inhomogeneous profile field and is well described by $B(z)=B_{p} \sqrt{1-z / z_{s}}$, except near its ends (see Fig. 2), where $z_{s}$ is the solenoid length and $z$ is the distance from the entrance of the solenoid. The thickness of these Zeeman compensating windings ranges from 28 layers at $z=0$ to zero layers at the end where $z=z_{s}$. Directly after the main solenoid we added the third set of windings composed of two small coils to tune the magnetic field in the extraction region [9]. These 4-cm-long coils are separated by only $1 \mathrm{~cm}$ and have an inner diameter of only $2.5 \mathrm{~cm}$ in order to be able to produce a large field gradient. Figure 2 shows the spatial dependence of the field. To prevent excessive heating of the solenoids we have placed water cooling on the inner and outer sides of the windings and between the two small coils.

The magnetic field and the polarization of the decelerating laser are chosen in such a way that the atoms are optically pumped into the highest hyperfine ground state $\left(F_{g}, M_{g}\right)$, which for $\mathrm{Na}$ is $\left(F_{g}=2, M_{g}=2\right)$. For the TOF technique we have added two laser beams labeled pump and probe as shown in Fig. 1. Because these beams cross the atomic beam at $90^{\circ}$ they excite atoms at all velocities. The probe beam has a diameter of $1 \mathrm{~mm}$, is located at a distance $z_{p}$ downstream from the pump beam, and its frequency is tuned to the strongest transition $\left(F_{g}=2 \rightarrow F_{e}=3\right)$ to maximize the fluorescence from the atomic beam. The fluorescence is collected on a photomultiplier tube. To enhance the collected fluores-

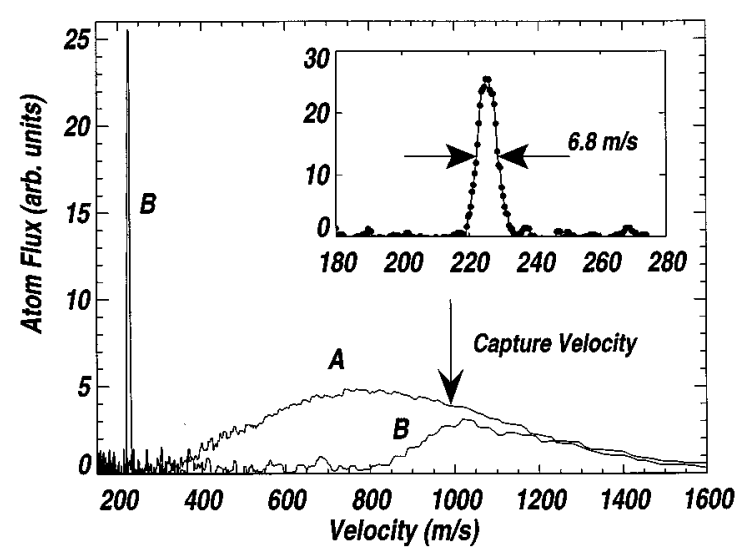

FIG. 3. Measured velocity distributions both with and without the slowing laser beam. The curve labeled $A$ indicates the unslowed distribution from the source. Curve $B$ shows the distribution after slowing. The arrow indicates the maximum of the velocity capture range of the slowing laser. The inset shows an enlarged portion of the figure in which the strong collection of atoms at low velocities is clearly visible.

cence, a spherical mirror is placed just below the atomic beam. The photomultiplier detects light in a region of $5 \mathrm{~mm}$, so the volume of atoms being probed is $\approx 5 \mathrm{~mm}^{3}$.

An AOM tunes the frequency of the pump beam $80 \mathrm{MHz}$ below that of the probe beam to optically pump the atoms from the $F_{g}=2$ to the $F_{g}=1$ ground state by exciting them to the nearly resonant $F_{e}=1$ and $F_{e}=2$ states. It transfers more than $98 \%$ of the population from $F_{g}=2$ to $F_{g}=1$ as the atoms pass through it $0.5 \mathrm{~mm}$ width. Since the $F_{g}=1$ atoms are too far out of resonance $(1772 \mathrm{MHz})$ with the probe beam, we do not observe fluorescence at the detector during the time the pump beam is on. Thus the pump beam acts as an optical shutter for the $F_{g}=2$ atoms.

In a similar manner we can use the pump beam as an optical shutter for atoms in the $F_{g}=1$ hyperfine ground state. To achieve this, we tune the probe laser frequency to the $F_{g}=1 \rightarrow F_{e}=0$ transition. Although weaker than the $F_{g}=2 \rightarrow F_{e}=3$ transition, the fluorescence from this transition is still strong enough to be detected in our experiment. We also rotated the AOM so that it upshifts the pump beam frequency to $+80 \mathrm{MHz}$ with respect to the probe beam. The pump beam frequency was thus just above the $F_{g}=1 \rightarrow F_{e}=2$ transition and it transfers atoms effectively from the $F_{g}=1$ to the $F_{g}=2$ ground state. It therefore serves as an optical shutter for the $F_{g}=1$ atoms.

To measure the velocity distribution of atoms in the $F_{g}=$ 2 ground hyperfine state we interrupt the pump laser beam for a period 10-50 $\mu$ s with the AOM. A pulse of atoms passes the pump region in the $F_{g}=2$ state and travels to the probe beam. The time dependence of the fluorescence induced by the probe laser gives the time of arrival, and this signal is readily converted to a velocity distribution. The deceleration laser beam is shut off during the free flight of the distance $z_{p} \approx 40 \mathrm{~cm}$ between the pump and probe regions to minimize the effects of off-resonance optical pumping or deceleration. Figure 3 shows the measured velocity distribution of the atoms emitted from the oven with and without the decelerating laser present.

In this method, the velocity resolution $\Delta v$ is not limited 
by the natural width of the excited state to $\Delta v=\Gamma / k(\approx 6$ $\mathrm{m} / \mathrm{s}$ ) as in previously described Doppler techniques that make use of the Doppler shifts of the moving atoms $[5,6,10]$. Instead, the limit is imposed by the duration of the pump laser gate $\Delta t$ and the diameter $d$ of the probe laser beam $(d \leqslant 1.0 \mathrm{~mm})$ to $\Delta v=v(v \Delta t+d) / z_{p}$, typically less than 1 $\mathrm{m} / \mathrm{s}$. Here $k \equiv 2 \pi / \lambda$ is the magnitude of the optical wave vector. This provides the capability of measuring the shape of the velocity distribution with resolution $\approx 10$ times better than $\Gamma / k$ as compared to the Doppler method. Furthermore, the resolution improves for decreasing velocity $v ; \Delta v$ is smaller than the Doppler cooling limit of $\sqrt{\hbar \Gamma / M_{\mathrm{Na}}} \approx 30$ $\mathrm{cm} / \mathrm{s}$ for $v \approx 80 \mathrm{~m} / \mathrm{s}$ and $\mathrm{Na}$ atoms.

\section{VELOCITY DISTRIBUTION OF THE SLOWED ATOMS}

\section{A. Model}

The deceleration process for the Zeeman technique is described by Bagnato et al. [8]. The force on the atoms is given by

$$
F(z, v)=\frac{\hbar k \Gamma}{2} \frac{s_{0}}{1+s_{0}+4\left[\delta+k v-\mu^{\prime} B(z) / \hbar\right]^{2} / \Gamma^{2}} .
$$

Here

$$
\mu^{\prime}=\mu_{B}\left[m_{e} g_{\left(3{ }^{2} P_{1 / 2}, F_{e}=3\right)}-m_{g} g_{\left(3{ }^{2} S_{1 / 2}, F_{g}=2\right)}\right]
$$

where $\mu_{B}$ is the Bohr magneton, the subscripts $g$ and $e$ refer to ground and excited states, $g_{\text {(level) }}$ is the Landé $g$ factor for that level, and $\delta=\omega_{\text {laser }}-\omega_{\text {atom }}$ is the detuning of the laser frequency $\omega_{\text {laser }}$ from the atomic transition frequency $\omega_{\text {atom }}$. Note that for $\mathrm{Na}$ we have $\mu^{\prime}=\mu_{B}$. The saturation parameter $s_{0}$ is given by $s_{0} \equiv I / I_{0}$, with $I$ the intensity of the slowing laser and $I_{0}$ the saturation intensity given by

$$
I_{0}=\frac{\Gamma \hbar \omega_{0}^{3}}{12 \pi c^{2}}
$$

For the $3{ }^{2} S_{1 / 2} \rightarrow 3{ }^{2} P_{3 / 2}$ transition of sodium, $I_{0}=6 \mathrm{~mW} /$ $\mathrm{cm}^{2}$. In the present discussion we will assume an ideal magnetic field

$$
B(z)=B_{b}+B_{p} \sqrt{1-z / z_{s}}
$$

Here $B_{p}=\hbar k v_{s} / \mu^{\prime}$ is related to the maximum velocity $v_{s}$ that can still be decelerated in the solenoid. Since the deceleration can never exceed $a_{D} \equiv \hbar k \Gamma / 2 M$, the minimum length of the solenoid is $z_{D} \equiv v_{s}^{2} / 2 a_{D}$ and we can define the fraction $\eta \equiv z_{D} / z_{s}$.

Equation (1) cannot be solved exactly; however, we can make an informative approximation by transforming the equation of motion for the atoms in the solenoid to a noninertial frame $\mathcal{R}$ that decelerates at $a=-\eta a_{D}$. The velocity is then given by $v_{\mathcal{R}}=v_{s} \sqrt{1-z / z_{s}}$. For this frame $\mathcal{R}$, the Doppler shift at the velocity $v_{\mathcal{R}}$ is just compensated by the Zeeman shift of the magnetic field profile, and one obtains for the velocity $v^{\prime}=v-v_{\mathcal{R}}$ measured in this frame

$$
\frac{d v^{\prime}}{d t}=-a_{D}\left(\frac{s_{0}}{1+s_{0}+4\left(\delta^{\prime}+k v^{\prime}\right)^{2} / \Gamma^{2}}-\eta\right)
$$

with $\delta^{\prime} \equiv \delta-\mu^{\prime} B_{b} / \hbar$. Here we assumed that $v^{\prime} \ll v_{\mathcal{R}}$. In the stationary situation $\left(d v^{\prime} / d t=0\right)$ we find for the equilibrium velocity $v_{\mathrm{eq}}^{\prime}$

$$
k v_{\mathrm{eq}}^{\prime}=-\delta^{\prime}-\frac{\Gamma}{2} \sqrt{\left(\frac{1-\eta}{\eta}\right) s_{0}-1},
$$

which is constant during the slowing process. So the atoms are surfing in this decelerating frame $\mathcal{R}$ at a constant velocity and at the end of the solenoid, where $v_{\mathcal{R}}=0$, the atoms can be extracted at their stationary velocity $v_{\text {eq }}^{\prime}$. Note that $\eta>s_{0} /\left(1+s_{0}\right)$ requires the acceleration to exceed $a_{\text {max }}=a_{D} s_{0} /\left(1+s_{0}\right)$, so that the square root remains real.

The atoms are not only decelerated in this frame at a constant velocity $v_{\text {eq }}^{\prime}$ but are also cooled towards this velocity [8]. We show this by linearizing the force in Eq. (5) around $v^{\prime}-v_{\text {eq }}^{\prime}$. We obtain $d\left(v^{\prime}-v_{\text {eq }}^{\prime}\right) / d t=\gamma\left(v^{\prime}-v_{\text {eq }}^{\prime}\right)$, where the damping rate $\gamma$ is

$$
\gamma=\frac{8 \gamma_{D} \eta^{2}}{s_{0}} \sqrt{\left(\frac{1-\eta}{\eta}\right) s_{0}-1}
$$

and

$$
\gamma_{D}=\frac{1}{4} \frac{\hbar k^{2}}{m}
$$

Here $\gamma_{D}$ is the maximum damping rate obtainable in Doppler cooling and we see that the damping rate in the Zeeman slowing process is just a fraction of $\gamma_{D}$. Furthermore, one observes that the damping rate becomes small for both small and large $s_{0}$, so for the optimum damping we have to choose $s_{0}=2$ and $\eta=0.5$.

The cooling during the slowing in the Zeeman technique has similarities with cooling in Doppler optical molasses. In Doppler molasses, the atoms are cooled towards zero velocity by the combined action of two counterpropagating laser beams. Atoms moving to the right are Doppler shifted closer to resonance with the laser beam coming from the right and out of resonance with the laser beam coming from the left and therefore are slowed down. The opposite holds for atoms moving to the left. In the Zeeman technique, where there is only one laser beam, this balancing between the laser beams is replaced by the action of the profile field, which tunes atoms moving too fast into resonance and atoms moving too slowly out of resonance. Since both the damping coefficient and the diffusion constants are comparable in both cases, one expects that the width of the velocity distribution in the Zeeman technique, in principle, will be comparable to the Doppler width $v_{D}=\sqrt{\hbar \Gamma / M}$.

One of the drawbacks of this treatment is that it is valid only as long as $v_{\mathrm{eq}}^{\prime} \ll v_{\mathcal{R}}$. Although this condition is fulfilled during most of the slowing process, it is no longer valid near the end of the solenoid, and this is just the position at where final velocity of the atoms is determined. 
It is important to realize that the ideal magnetic field does not even produce a constant deceleration [7]. This is easily seen by differentiating the resonance condition

$$
k v=\omega_{b}+\omega_{p} \sqrt{\widetilde{z}}-\delta=\omega_{p} \sqrt{\widetilde{z}}-\delta^{\prime},
$$

where $\omega_{b, p}=\mu^{\prime} B_{b, p} / \hbar$ and $\tilde{z} \equiv\left(1-z / z_{s}\right)$. The deceleration satisfying the resonance condition is then

$$
a=-\eta a_{D}\left(1-\frac{\delta^{\prime}}{\omega_{p} \sqrt{\widetilde{z}}}\right)
$$

using $(d / d t) \sqrt{\widetilde{z}}=v(d / d z) \sqrt{\widetilde{z}}$. In the usual operating condition to extract slow atoms from the solenoid, $\delta^{\prime}<0$ [see Eq. (6)]. Then $\widetilde{z}$ eventually becomes small enough that maintaining the resonance condition requires the magnitude of the deceleration to exceed $a_{\max }$. The atoms then drop out of the process and emerge from the solenoid as desired. Only for $\delta^{\prime}=0$ is the deceleration constant.

\section{B. Experimental results}

From the measured velocity distribution of Fig. 3 we can obtain three quantities: the central velocity, the width, and the peak area. In Fig. 4 we have plotted these quantities as a function of the slowing laser intensity. For the central velocity we see that it varies approximately with the square root of the intensity [dashed line in Fig. 4(a)], in agreement with the model presented in Sec. III A. However, the width of the distribution is much larger than the expected Doppler width $v_{D} \approx 30 \mathrm{~cm} / \mathrm{s}$. Also there is a strong intensity dependence of the width, which is not the case for a Doppler cooling process.

In order to understand these measurements we have made a numerical simulation of the deceleration process, where we numerically integrated the equation of motion of the atoms to find the final velocity at the exit of the solenoid. In this way we avoid the approximations we had to make in order to obtain the analytical results of Sec. III A. At the same time we can account for the real magnetic field, as plotted in Fig. 2. Furthermore, this allowed us to account for the intensity profile of the Gaussian laser beam since both the equilibrium velocity $v_{\text {eq }}^{\prime}$ and the drop out condition $a>a_{\max }$ depend on the intensity.

The numerical integration to calculate the atomic trajectories was done for several values of $s_{0}$ to find the intensity dependence of the final velocity $v_{f}\left(s_{0}\right)$. In order to compare this result with the experimental results, we calculate a weighted average of $v_{f}\left(s_{0}\right)$ over the intensity profile of the slowing beam. To find the weighting function, we make the transformation of the radial intensity profile $s_{0}(\rho)$ of the slowing beam to an area intensity function $F\left(s_{0}\right)$ such that $F\left(s_{0}\right) d s_{0}$ is proportional to the area of the slowing beam with intensity between $s_{0}$ and $s_{0}+\mathrm{d} s_{0}$. For a Gaussian intensity profile with a beam waist $w$ we find $F\left(s_{0}\right)=\pi w^{2} / s_{0}$ for $0 \leqslant s_{0} \leqslant s_{0}^{\max }$ and $s_{0}^{\max }$ is the maximum intensity in the center of the laser beam. In our calculations, the lower limit of $s_{0}$ is the intensity at the edge of the beam where it is cut off by an aperture. Using the calculated intensity dependence of the final velocity $v_{f}\left(s_{0}\right)$ and the calculated area intensity function $F\left(s_{0}\right)$, it was straightforward to determine the laser in-
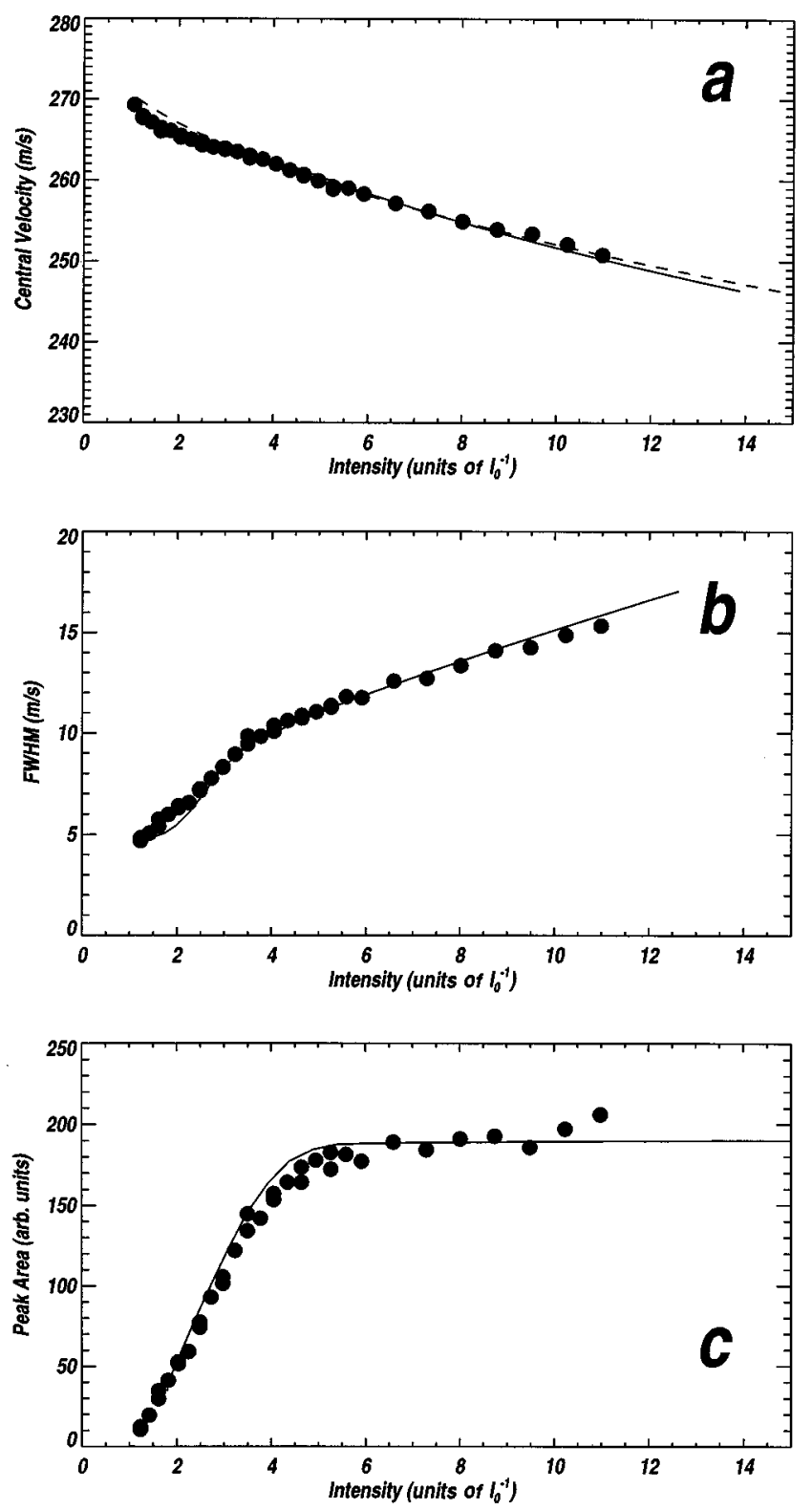

FIG. 4. Intensity dependence for a fixed laser frequency for the three quantities: (a) central velocity, (b) width of the velocity distribution, and (c) the peak area. The dots indicate the measured data points. The solid lines represent the calculated intensity dependence, using a Gaussian slowing beam profile. The dashed line indicates the result of Eq. (6). The intensity is determined at the end of the solenoid and is given in units of the saturation intensity $I_{0}$.

tensity dependence of the width, peak area, and center of the final velocity distribution.

Our calculations are presented together with the measurements in Fig. 4. The agreement between the calculations and the experimental data is quite good; only at low intensities some minor discrepancies are found. The reason for these discrepancies might be found in the choice of a Gaussian slowing beam profile. Calculations with different intensity distributions might result in even better agreement.

Given this sensitivity of the properties of the slowed atom distribution to the intensity profile of the slowing laser beam, we changed our optics such that the slowing laser beam profile was flatter, by accepting only its central part with a di- 


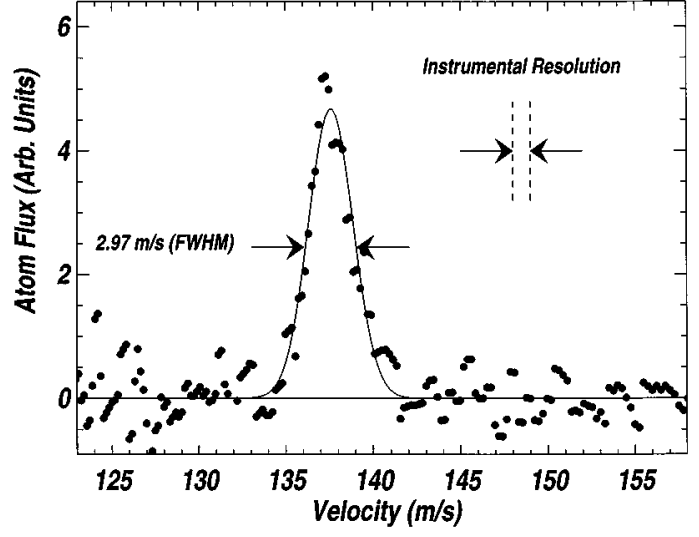

FIG. 5. One of the measured velocity distributions with the flattened laser beam profile. The experimental width of approximately $1 / 6(\Gamma / k)$ is shown by the dashed vertical lines between the arrows. The Gaussian fit through the data yields a FWHM of $2.97 \mathrm{~m} / \mathrm{s}$.

ameter of $6 \mathrm{~mm}$ after expanding it with a telescope. This led to a drop in available slowing power, so that measurements could be performed only at relatively low intensity. Although the Gaussian character of the slowing beam was flattened out significantly, small optical imperfections still resulted in residual spatial intensity variations. These variations had 5\% rms scatter (measured outside of the vacuum system) and the area intensity function $F\left(s_{0}\right)$ had an approximately Gaussian character centered at the average intensity.

Figure 5 shows the final velocity distribution for one of the measurements with this much flatter beam intensity profile, giving a full width at half maximum (FWHM) of $3.0 \mathrm{~m} / \mathrm{s}$ at a central velocity of $138 \mathrm{~m} / \mathrm{s}$. We note that the width is about one-half of $\Gamma / k$; however, it is still 4 times larger than the Doppler limit. Our measurements of the intensity dependence of the width of the velocity distribution using the flatter profile (Fig. 6) show a much weaker increase with increasing laser intensity than Fig. 4. This can be fitted with

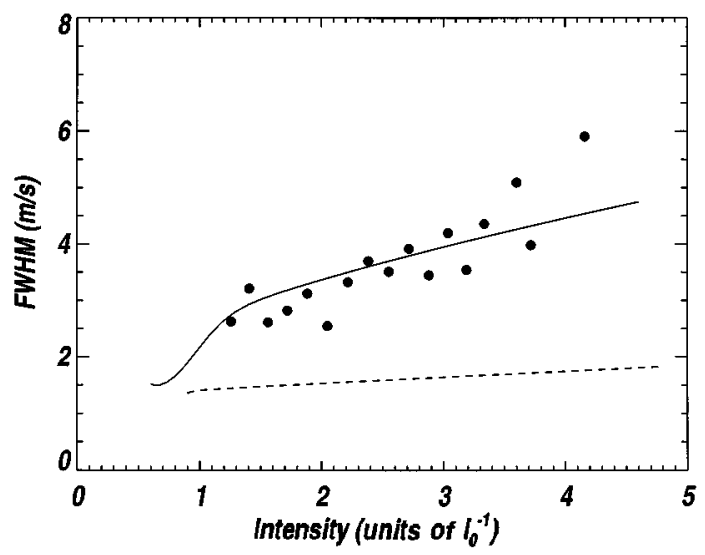

FIG. 6. Intensity dependence of the width of the cold atom peak for the spatially expanded slowing beam. On the horizontal axis the average intensity of the slowing beam is represented. For the calculations we assumed the area intensity function $F\left(s_{0}\right)$ to be Gaussian. The dashed curve gives the calculated result for the experimentally determined distribution with rms fluctuations of 5\%; the solid line gives a fit to the data, using an intensity distribution function with rms fluctuations of $18 \%$.
TABLE I. Some of the residual velocity-broadening mechanisms for atoms with a velocity of $140 \mathrm{~m} / \mathrm{s}$. These effects lead to a minimum width on the order of $1.6 \mathrm{~m} / \mathrm{s}$.

\begin{tabular}{lc}
\hline \hline Effect & Width $(\mathrm{m} / \mathrm{s})$ \\
\hline finite gate time $(10 \mu \mathrm{s})$ & 0.42 \\
finite gate width $(0.5 \mathrm{~mm})$ & 0.16 \\
residual magnetic-field inhomogeneities & 0.8 \\
spectral distribution of the slowing beam & 0.6 \\
diffusion due to spontaneous emission & 1.2 \\
\hline \hline
\end{tabular}

the calculated intensity dependence convolved with the flattened slowing beam area intensity function $F\left(s_{0}\right)$ having a rms width of about $18 \%$ (shown by the solid line in Fig. 6), but not by an intensity dependence convolved with an area intensity function having the measured $5 \%$ width discussed above (dashed line). This difference may possibly result from further variations in the intensity caused by passage of the beam through the window of the vacuum system that could produce distortions and interference patterns.

There are other broadening mechanisms that we did not take into account in the previous discussion (see Table I). We neglected the broadening of the slowed atom velocity distribution caused by the heating resulting from the random direction of the spontaneously emitted photons. Widths resulting from this heating lie on the order of $1.2 \mathrm{~m} / \mathrm{s}$, which is typical for a Doppler cooling process. Another broadening mechanism derives from the spectral distribution of the slowing laser of approximately $\Delta \nu_{c}=1.0 \mathrm{MHz}$. Since the final velocity of the atoms that emerge from the Zeeman slower is directly related to the frequency of the laser [see Eq. (6)], fluctuations in the slowing laser frequency will directly affect the final velocity distribution. The resulting broadening is calculated using $\Delta v_{f}=\Delta \nu_{c} \lambda \approx 0.6 \mathrm{~m} / \mathrm{s}$. Further, there are effects from changes of the magnetic field at locations displaced from the symmetry axis of the magnet. Near the point where the extraction takes place, the axial field curvature in the $z$ component of the magnetic field $B_{z}$ is $1 / 2\left(d^{2} B_{z} / d z^{2}\right) \approx 25 \mathrm{G} / \mathrm{cm}^{2}$. From an expansion of the field near this point [11] we obtain to second order the radial curvature of $B_{z}: \quad 1 / 2\left(d^{2} B_{z} / d \rho^{2}\right)=-1 / 4\left(d^{2} B_{z} / d z^{2}\right) \approx$ $-12.5 \mathrm{G} / \mathrm{cm}^{2}$. (The radial component $B_{\rho}$ is zero to second order.) Therefore atoms that propagate at the edges of the slowing laser beam (with radius $3.0 \mathrm{~mm}$ ) experience a magnetic field that is $\Delta B=1.1 \mathrm{G}$ smaller than at the center. Consequently, there will be a spread $\Delta v_{f}=\mu^{\prime} \Delta B / \hbar k \approx 0.8$ $\mathrm{m} / \mathrm{s}$ in velocity resulting from this $\Delta B$. All of these broadening mechanisms add to the velocity spread of the atoms emerging from the Zeeman slower. Consequently, the minimum achievable width in our experiment is around $1.6 \mathrm{~m} / \mathrm{s}$. The minimum widths of the velocity distributions that we measured in the experiments $(\approx 2.5 \mathrm{~m} / \mathrm{s})$ are therefore still dominated by the intensity inhomogeneities in the laser beam.

\section{MAPPING OF THE PHASE SPACE}

The different and important capability of our apparatus is a very much more powerful and informative scheme of data acquisition that results from shutting off the slowing laser 

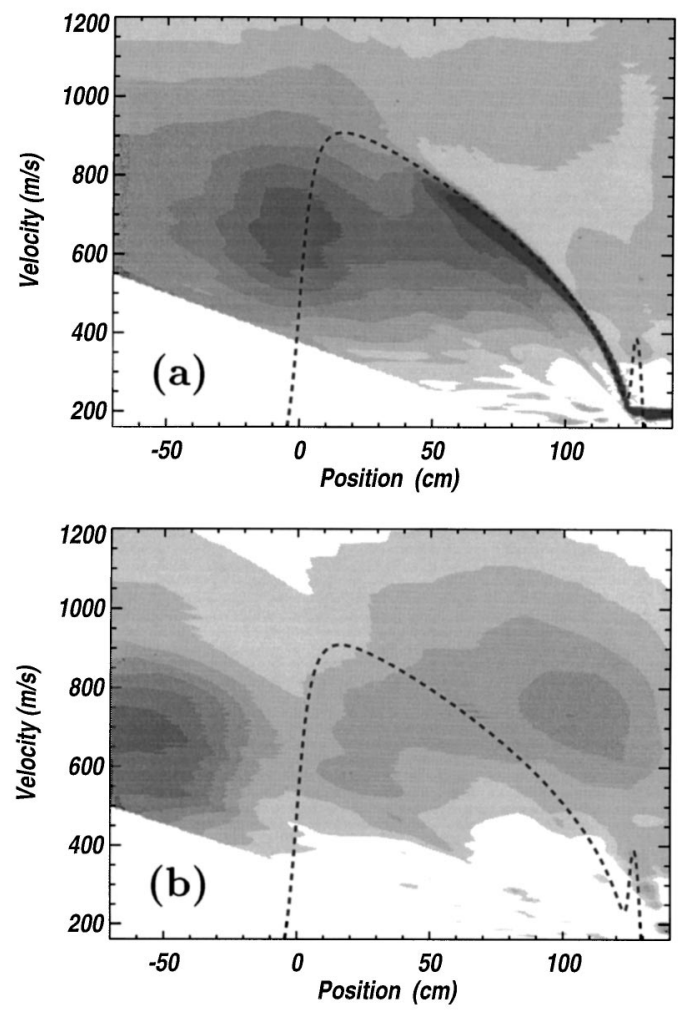

FIG. 7. Contour map of the measured velocity and position of atoms in the solenoid for (a) $F_{g}=2$ atoms and (b) $F_{g}=1$ atoms. The dashed line indicates the resonance frequency for the $(F$, $\left.M_{F}\right)=(2,2) \rightarrow(3,3)$ cooling transition. The density of atoms per unit phase-space area $\Delta v \Delta z$ has been indicated with different gray levels.

beam a variable time $\tau$ before the short shutoff of the pump beam. Then the atoms that pass through the pump region during the short time when the pump beam is off already have traveled a distance $\Delta z=v(z) \tau$ [at constant velocity $v(z)$ because the slowing laser was off] and their time of arrival at the probe laser is $z_{p} / v(z)=z_{p} \tau / \Delta z$. Thus the TOF signal contains information not only about the velocity of the detected atoms, but also about their position $z\left(=z_{s}+z_{c}\right.$ $-\Delta z)$ in the magnet at the time the slowing laser light was shut off. (Here $z_{c} \approx 25 \mathrm{~cm}$ is the distance from the end of the solenoid to the position where the pump beam is placed.) Since the spatial dependence of the magnetic field is known (Fig. 2), both the field and atomic velocity at that position can be determined and the TOF signal is proportional to the number of atoms in that particular region of phase space. This technique therefore gives a mapping of the atomic population in the two-dimensional phase space within the solenoid.

Such a mapping of the velocity distribution within the solenoid is a powerful diagnostic of the Zeeman compensating scheme for atomic beam deceleration. The contours of Figs. 7(a) and 7(b) represent the strength of the TOF signal for each of the two ground-state hyperfine levels, and thus the density of atoms, at each velocity and position in the magnet. The dashed line shows the velocity $v(z)=\left[\mu^{\prime} B(z) / \hbar-\delta\right] / k$ for which the magnetic field tunes the atomic transition $\left(F, m_{F}\right)=(2,2) \rightarrow(3,3)$ into resonance with the decelerating beam.
There is much to be learned from Fig. 7. The most obvious information in Fig. 7(a) is that atoms are strongly concentrated at velocities just below that of the resonance condition, as predicted by Eq. (6). This corresponds to the strong peak of slow atoms shown in Fig. 3. The buildup of atoms in this region of phase space is indeed strong and begins even at very high speeds. However, at the same time it is evident that the width of the velocity distribution is already large during the slowing process, i.e., that the final width of the velocity distribution is not caused by the extraction of the atoms from the slowing process. This is consistent with our claim that the width is determined mainly by the inhomogeneity in the laser beam.

\section{A. Optical pumping}

We note the strong buildup of $F_{g}=2$ atoms over a wide velocity range centered near $700 \mathrm{~m} / \mathrm{s}$ at the entrance of the solenoid, as shown in Fig. 7(a), and a corresponding depletion of $F_{g}=1$ atoms, as shown in Fig. 7(b). This strong optical pumping occurs between the oven and the magnet where the slowing laser light is very intense because it is focused on the oven. For typical values of $30 \mathrm{~mW}$ laser power and a beam diameter of $\approx 100 \mu \mathrm{m}$, the intensity of $3 \times 10^{5} \mathrm{~mW} / \mathrm{cm}^{2}$ broadens the absorption line from its natural width of $10 \mathrm{MHz}$ to about $2 \mathrm{GHz}$, more than enough to compensate the Doppler shift of the entire velocity distribution. Since the detuning is $50 \mathrm{MHz}$ above resonance for the $F_{g}=2 \rightarrow F_{e}=3$ transition, the optical pumping is most effective on $F_{g}=1$ atoms, Doppler shifted by their velocities near $1000 \mathrm{~m} / \mathrm{s}$, not far from the peak of the distribution at $700 \mathrm{~m} / \mathrm{s}$. Of course, atoms in $F_{g}=2$ can also be excited, but they are farther from resonance, so that the net transfer of population is from $F_{g}=1 \rightarrow F_{g}=2$.

As the atoms enter the solenoid this effect is partially reversed. Figure 7(a) shows a decrease of the $F_{g}=2$ population near $700 \mathrm{~m} / \mathrm{s}$ in the rising edge of the magnetic field, while Fig. 7(b) shows a corresponding increase of $F_{g}=1$ atoms. In this field region, the excitations from the $F_{g}=2$, $m_{g}=-1,0$, and +1 levels to the states appropriate for circularly polarized $\left(\sigma^{+}\right)$light $\left(\Delta m_{F}=+1\right)$ come into resonance with the laser (see Fig. 8). In the relatively large magnetic field in this region $(400<B<1100 \mathrm{G})$ the excited eigenstates have changed because of the hyperfine uncoupling in such a way that the spontaneous decay from those states goes predominantly via $\Delta m_{F}=-1$. The atoms therefore do not gain angular momentum in absorption-decay cycles and do not reach the slowing transition $\left(F, m_{F}\right)(2,2)$ $\rightarrow(3,3)$, but instead stay trapped in one of the $F_{g}=1$ states, which are far from resonance. Similar optical pumping schemes can be used to explain the appearance and disappearance of other population islands in Fig. 7, as well as in other data sets taken with different laser detunings.

\section{B. Extraction coils}

One of the main advantages of the Zeeman compensation method over others is the production of a continuous beam of slow atoms and the deceleration of almost all the atoms emitted by the source. This efficiency is compromised, however, by the further deceleration and broadening of the velocity distribution as the atoms travel outside of the magnet. 


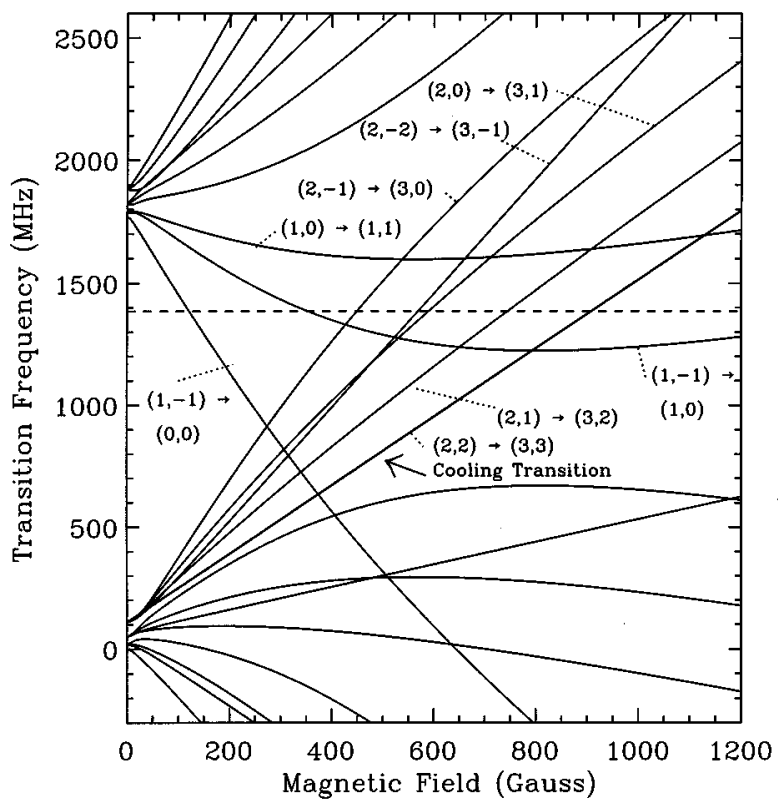

FIG. 8. Plot of optical transition frequencies vs magnetic field for $\sigma^{+}$light. The labels at the lines represent the atomic transitions in the limit for $B \rightarrow 0$ in the notation $\left(F_{g}, M_{g}\right) \rightarrow\left(F_{e}, M_{e}\right)$. The arrow indicates the cycling transition $\left(F, M_{F}\right)=(2,2) \rightarrow(3,3)$ used for slowing. The frequency scale is with respect to the zerofield value of the $F_{g}=2 \rightarrow F_{e}=0$ transition. The dashed line indicates the Doppler-shifted frequency of the slowing laser for atoms moving at $700 \mathrm{~m} / \mathrm{s}$ and a laser frequency tuned $80 \mathrm{MHz}$ above $F_{g}=$ $2 \rightarrow F_{e}=3$. In the rising edge of the magnetic field the atoms first come into resonance with the other $m_{g} \rightarrow M_{e}$ transitions originating from $F_{g}=2 \rightarrow F_{e}=3$.

Shutting off the slowing beam ameliorates this problem of course, but the price is the loss of the advantages cited above. In order to preserve both of these advantages, we have placed additional coils at the end of the solenoid to tailor the field profile and thereby enhance the extraction of the atoms from the solenoid. The position of these coils is shown in Fig. 1 and their effect on the axial magnetic field profile is shown in Fig. 2.

The function of the extraction coils is to give a strong detuning to the atoms from the slowing laser when they come to the end of the main solenoid. The first one of the extraction coils increases the magnetic field sharply to accomplish the detuning. The atoms then stop absorbing light and propagate freely. The reduction of the field to zero is done by the second of the small coils, which generates the same magnetic field, but in the opposite direction, by applying a reverse current. This produces a large magnetic-field gradient between the two coils, which prevents the atoms from scattering much light when they pass through resonance. Both the measured and the calculated drop in velocity at this point are small enough to be neglected. Such an extraction scheme is similar to the $\sigma^{-}$-slowing scheme [7], where the atoms are extracted from the maximum in the magnetic field rather than in the minimum field.

\section{TWO-VELOCITY BEAM}

In collision physics much is to be learned from the study of collision cross sections over a broad velocity range. Study

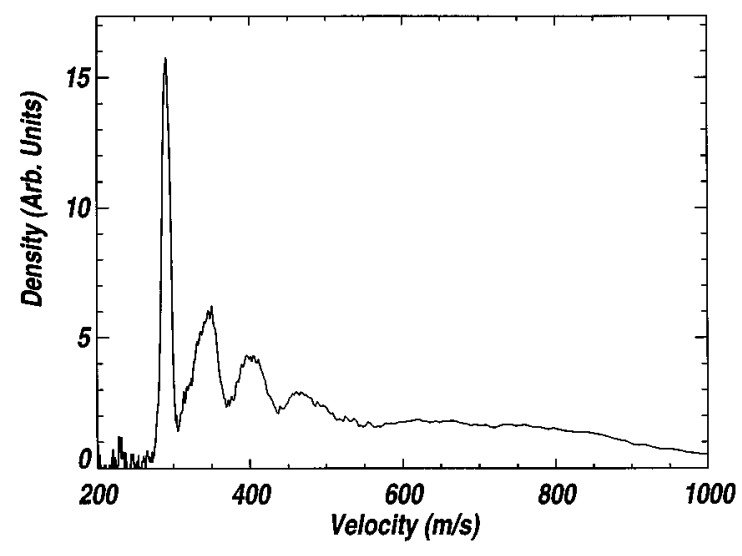

FIG. 9. Measured velocity distribution with the auxiliary coils both operating at positive current. The figure clearly shows the multiple peaks that arise from them.

of collisions in a trap probe the region around $1 \mathrm{~m} / \mathrm{s}$ and allows little variation in velocities since increasing the temperature in a trap decreases the density. Study of collisions resulting from crossing a MOT with a cold atomic beam yields information in the range $100-400 \mathrm{~m} / \mathrm{s}$. Information in the intermediate range $(1-100 \mathrm{~m} / \mathrm{s})$ can be obtained if one is able to generate two velocity classes in one single beam and study head-tail collisions [12]. In this way the velocity of the atoms can still remain large $(200 \mathrm{~m} / \mathrm{s})$, so that the transverse spreading remains small. The only requisite to achieve this is the ability to generate two, well-defined velocity classes in one beam.

We have made an attempt to study such a possibility by using two extraction coils, as shown in Fig. 1. When tuning the current through these coils we have found unexpected features that could not be explained by our careful study of optical pumping effects, magnetic-field imperfections, magnetic forces, or other phenomena. These features manifested themselves as multiple peaks, approximately equally spaced, as shown in Fig. 9. We speculated that they arose from atoms that had dropped out of resonance all along the beam path because they were near the edge of the Gaussian laser beam and are thus subject to lower intensity than those at the center. Such atoms were lost to the main deceleration process, but may have undergone a little further deceleration as they passed through the field of the extraction coils.

The speculation mentioned above about the source of the additional peaks in Fig. 9 is clearly supported by both the measurements shown in Fig. 10(a) and our simulations presented in Fig. 10(b). In the measurement we deliberately misaligned the slowing beam so that there is a wide range of intensities within its diameter. Furthermore, we reversed the current through the second extraction coil to demonstrate clearly the additional slowing of the atoms that have dropped out earlier in the deceleration process. Figure 10(a) clearly shows that atoms begin dropping out in substantial numbers at $z=110 \mathrm{~cm}$ and that this dropout finds its maximum near $z=119 \mathrm{~cm}$ where the magnetic field attains its maximum gradient $(23 \mathrm{G} / \mathrm{cm})$. After this point the dropout rate decreases as the magnetic field gradient decreases. The remaining atoms then decouple from the field at $z=123 \mathrm{~cm}$ where it has decreased to its minimum of $320 \mathrm{G}$. Those atoms lose some more velocity when they come into resonance between 

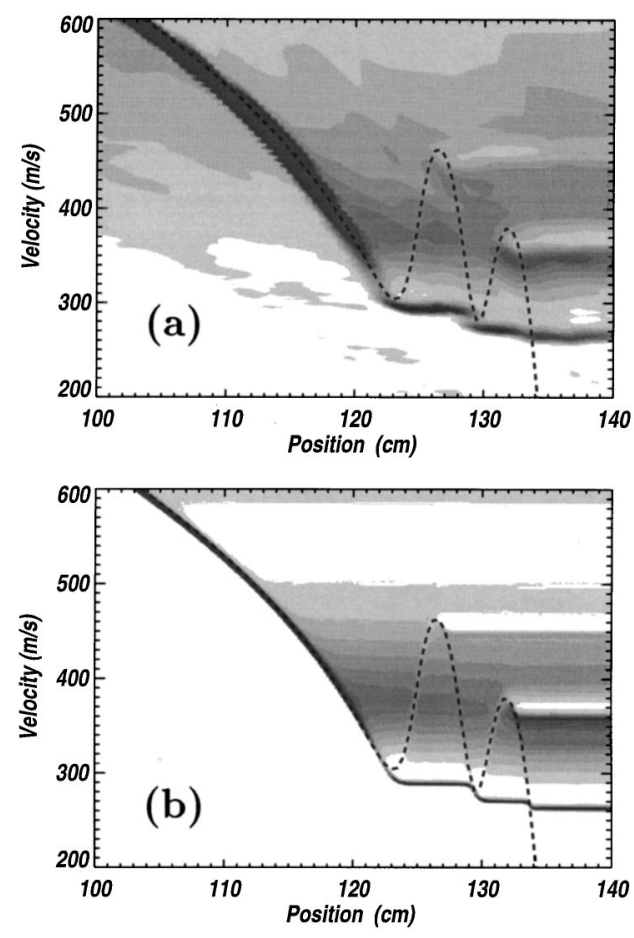

FIG. 10. Contour map of the velocity and position of atoms in the solenoid showing the origin of the multiple peaks structures visible in Fig. 9. (a) shows the measured results and (b) shows the result of the simulations discussed in the text. The dashed lines show the velocity for the atoms in resonance with the cooling transition $\left(F, M_{F}\right)=(2,2) \rightarrow(3,3)$.

the two extraction coils $(z=129 \mathrm{~cm})$, but the velocity change at the passage through resonance at $z=133 \mathrm{~cm}$ is negligible because the field gradient is so large.

Some of the atoms that had dropped out earlier in the slowing process come into resonance with the magnetic field again at the extraction coils. Near the field maxima of those coils, the field gradient is small enough for the atoms to remain resonant for a relatively long distance and they therefore experience a large drop in velocity. This results in the depleted areas in phase space just downstream near the maxima of the field from the extraction coils. Compensating areas of increased density appear just below the maxima of those extraction coils as can be seen in Fig. 10. Those structures are projected as multiple peaks in the velocity distribution from the Zeeman slowing process as shown in Fig. 9.

Our simulations for this additional slowing effect are shown in Fig. 10(b). We calculated the atomic trajectories for a distribution of intensities within the slowing beam. We used the force given by Eq. (1) to calculate the trajectories of the atoms. We averaged these trajectories assuming a Gaussian laser area intensity function $F\left(s_{0}\right)$, which is centered at the average $\overline{s_{0}}=3.5$ and having a width $\Delta s_{0}=3.0$. As can been seen by comparing Figs. 10(a) and 10(b) the simulations contain all the essential features of the experiments.

In principle, the effect that produces the multiple velocity peaks could be used more systematically to generate two specific final velocities with a selectable velocity difference. By tuning this difference between the two groups, we could have a superb tool for the study of head-tail collisions. We would let half of the atoms drop out of the slowing process

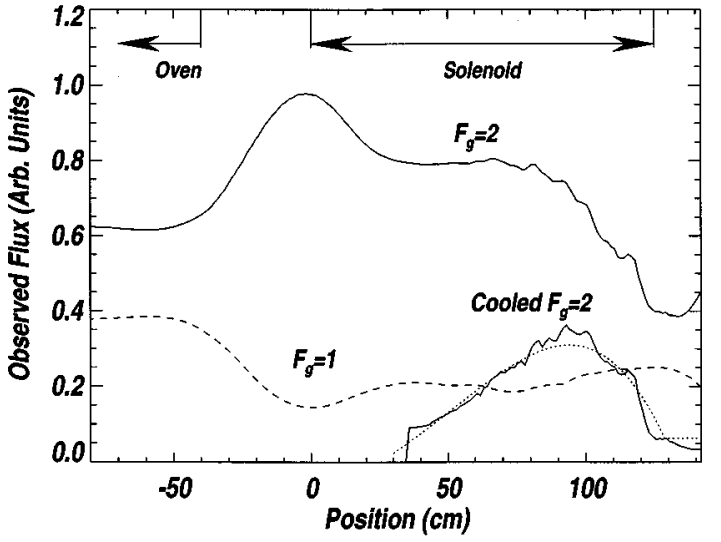

FIG. 11. Atom flux observed at the detector for atoms coming from different positions in the solenoid for both ground hyperfine states. The solid line on the lower right-hand side of the figure represents the measured flux of laser-cooled atoms in the $F_{g}=2$ state. The dotted line is the result of the calculations discussed in the text.

as in Fig. 10, while the other half is slowed in the conventional way. After this first slowing stage we would put a second, short Zeeman slower for the atoms that have dropped out, producing an effect similar to shown in Fig. 10(a). The field tuning of this second Zeeman slower can be used to tune the final velocity of those atoms independent of the other atoms that are far off-resonance at this point.

\section{ATOM FLUX}

One of the important parameters in collision physics using beam techniques is the flux of atoms. The signal in a collision experiment is directly proportional to the flux and in the case of studying collision within one beam the signal depends quadratically on the flux. In a slowing process of atoms having a hyperfine structure (such as sodium), loss of atoms can be caused among other things by two mechanisms: (i) Atoms can fail to be kept resonant with the laser and (ii) atoms are pumped to the wrong hyperfine state, i.e., the state not coupled to the slowing laser. In a conventional technique of measuring the velocity distribution, the effect in both cases would be a small signal. Furthermore, it is not possible to determine the cause of the loss of signal. Since the conventional techniques do not allow detection of the loss during the slowing process, it is hard to make an assessment of the problem.

The technique reported here enables us to monitor the atom flux in each ground hyperfine state throughout the slowing process as shown in Fig. 11. The flux of slowed atoms, originating from a certain (variable) position $z$, can be separated from the total atom flux, by taking the atoms within a narrow velocity range around the locally resonant velocity. This gives an indication of the number of atoms slowed down during the entire process.

To relate the flux for the $F_{g}=1$ and $F_{g}=2$ ground states to each other we assumed that at the left edge of Fig. $11(z$ $<-40 \mathrm{~cm}$ ) the atoms leave the oven with the expected statistical weights of $3 / 8$ and $5 / 8$ for the $F_{g}=1$ and $F_{g}=2$ states. This is a reasonable assumption since atoms that ap- 
pear to come from positions $z<-40 \mathrm{~cm}$ emerged from the oven after the slowing beam was shut off. Consequently, one does not expect a deviation of the statistical weights of the ground-state populations.

Directly after the atoms leave the oven $(z=-40 \mathrm{~cm})$, a transfer from the $F_{g}=1$ to the $F_{g}=2$ state takes place, caused by the high intensities of the focused slowing beam in this region. Some of the atoms are optically pumped back to the $F_{g}=1$ state in the rising part of the magnetic field $(\mathrm{z}=$ $10 \mathrm{~cm}$ ), where the $F_{g}=2, m_{g}=-1,0$, and +1 states successively come into resonance with the light (see Fig. 8).

The collection of slowed atoms starts at $\mathrm{z}=+40 \mathrm{~cm}$, where the fastest atoms come into resonance with the slowing light and start to be decelerated as indicated near the bottom right of Fig. 11. The flux of atoms in the cold peak increases from this point since in the decreasing magnetic field atoms with lower initial velocities also come into resonance with the light. The maximum flux observed in this slowed peak is found at $z=95 \mathrm{~cm}$, where the decelerated atoms have a longitudinal velocity $v_{z}$ of $550 \mathrm{~m} / \mathrm{s}$. At this point $35 \%$ of the atoms emerging from the source in the direction of the detector are collected in the narrow peak of cooled atoms and reach the detector. After $z=95 \mathrm{~cm}$, the decrease of the longitudinal velocity of the atoms and the increase of the transverse spreading of the slowed beam caused by the transverse heating resulting from repeated spontaneous emissions starts to reduce the observed flux of slowed atoms at the detector, which is placed at $z=190 \mathrm{~cm}$. This continuous decrease of the observed flux at the detector stops when the deceleration and the transverse heating of the atoms stops near the extraction coils. So from the position of the extraction coils, the flux observed at the detector remains constant since both the transverse and the longitudinal velocity remain constant.

We made a model, based on geometric considerations, to verify the apparent decrease of the slowed atom flux on the detector due to longitudinal deceleration and the transverse spreading of the beam. Atoms decelerated in the Zeeman slower are slowed primarily in the longitudinal direction ( $v_{\|}$of the velocity). The transverse component $v_{\perp}$ increases at the same time because of repeated spontaneous emission of light in random directions. Thus the divergence of the atomic beam increases, leading to a drop of atom flux at the detector. The width of the final transverse velocity distribution $\delta v_{\perp}^{\prime}$ resulting from the scattering of $\bar{N}$ photons is given by $[13]$

$$
\delta v_{\perp}^{\prime}=\sqrt{\alpha v_{r}^{2} \bar{N}}
$$

with $v_{r}$ the recoil velocity $(3 \mathrm{~cm} / \mathrm{s}$ for sodium) and $\alpha$ a factor to account for the projection of the recoil of the spontaneously emitted photons on the transverse directions. For isotropic radiation we have $\alpha=1 / 3$ and for a dipole pattern directed along the longitudinal axis we have $\alpha=3 / 10$.

The total transverse velocity spread at a given position $z$ is obtained from

$$
\delta v_{\perp}=\sqrt{\left(\delta v_{i, \perp}\right)^{2}+\left(\delta v_{\perp}^{\prime}\right)^{2}}
$$

with $\delta v_{i, \perp}$ the initial transverse velocity spread arising from the finite collimation of the source. In our model we neglect the transverse width of the atomic beam during the slowing process. The geometrical probability $P_{\text {geo }}$ for the atoms at $z$ to be detected at $z_{d}$ is given by

$$
P_{\mathrm{geo}} \sim\left[\frac{v_{\|} / \delta v_{\perp}}{z-z_{d}}\right]^{2}
$$

The flux contributions from a given point in the Zeeman slower can be obtained by multiplying this geometrical probability $P_{\text {geo }}$ by the calculated number of atoms that is collected from the source distribution at this point. Figure 11 shows the result of this calculation combined with the experimentally observed slowed atom flux. Clearly, the result of our model agrees quite well with the measurements.

Finally, we have made also two independent estimates for the total flux of slowed atoms. Since there is transverse spreading of the slowed beam, estimating the total flux from the peak intensity in Fig. 3 and a detector efficiency is not easily done. First, we used the flux from the source calculated from its temperature and nozzle size. In a typical setup this was $\pm 10^{17}$ atoms $\mathrm{sr}^{-1} \mathrm{~s}^{-1}$. In the angular width of the slowing laser beam $(\approx 1 \mathrm{mrad})$ we therefore had a flux of approximately $5 \times 10^{11}$ atoms s $^{-1}$.

This is corroborated by a second independent estimate using the loss of slowing light flux. Though weak, the fluorescence from background sodium atoms near the oven could be measured. When we slowed the atoms with $P=30 \mathrm{~mW}$ of laser power, we lost approximately $\xi=20 \%$ of the incident light, compared to the situation of no slowing. If we assume an average atom to be slowed by $\Delta v_{\|} \approx 500 \mathrm{~m} / \mathrm{s}$, it must have scattered $\bar{N}=1.7 \times 10^{4}$ photons since $v_{r}=3$ $\mathrm{cm} / \mathrm{s}$. Thus we expect a slow atom flux $\mathcal{F}=\xi P / \bar{N} \hbar \omega=1.1$ $\times 10^{12}$ atoms $\mathrm{s}^{-1}$. From both estimates we conclude that the slow atom flux $\mathcal{F}$ is approximately $10^{12}$ atoms s $^{-1}$, in good agreement with the numbers presented by other groups [13]. Using optical compression in the final stage to condense the slowed beam to a diameter of $0.1 \mathrm{~mm}$ would result in a density of $10^{16}$ atoms $/ \mathrm{cm}^{2} \mathrm{~s}$, more than sufficient for many collision experiments.

\section{SUMMARY}

In conclusion, we have presented an alternative diagnostic technique for the study of the slowing process using Zeeman compensation. It enables us to obtain complete information on the velocity and position of the atoms during the slowing process without requiring complicated devices. This technique is hyperfine state selective, so that we obtain information about both the $F_{g}=2$ and $F_{g}=1$ ground states. Two conspicuous features can be distinguished in the measurements. First, we see a strong buildup of atomic phase-space density just below the velocities where the atoms are in resonance with the magnetic field. Second, we see a transfer of atomic population from one hyperfine state to the other at specific places in the solenoid, which indicates optical pumping effects in the local magnetic field.

With our hyperfine selective TOF method we were able to measure the longitudinal velocity spread of the atoms with a resolution that is better than in all previous works on the Zeeman slower. The resolution of those other techniques is limited to $\Delta v=\Gamma / k \approx 6 \mathrm{~m} / \mathrm{s}$, whereas our technique has an 
intrinsic resolution better than the Doppler limit of $30 \mathrm{~cm} / \mathrm{s}$ and improves with decreasing velocity. The velocity spread for the slowed atoms was caused mainly by intensity inhomogeneities of the slowing laser beam. When we changed the setup so that the intensity inhomogeneities in this beam were reduced, we observed widths down to $2.5 \mathrm{~m} / \mathrm{s}$. A further reduction of the width could not be realized, due mainly to residual inhomogeneities in the slowing laser beam.

\section{ACKNOWLEDGMENTS}

H.M. acknowledges support from the Dutch NWO, the NSF, and ONR. P.v.d.S. is financially supported by the Royal Dutch Academy of Sciences (KNAW).

Lafyatis, Phys. Rev. Lett. 67, 3483 (1991).

[8] V.S. Bagnato, A. Aspect, and S.C. Zilio, Opt. Commun. 72, 76 (1989).

[9] M.E. Firmino, C.A. Faria Leite, S.C. Zilio, and V.S. Bagnato, Phys. Rev. A 41, 4070 (1990).

[10] J. Prodan, A. Migdal, W.D. Phillips, I. So, H.J. Metcalf, and J. Dalibard, Phys. Rev. Lett. 54, 992 (1985).

[11] T. Bergeman, G. Erez, and H.J. Metcalf, Phys. Rev. A 35, 1535 (1987).

[12] Y. Wang and J. Weiner, Phys. Rev. A 42, R675 (1990).

[13] M.A. Joffe, W. Ketterle, A. Martin, and D.E. Pritchard, J. Opt. Soc. Am. B 10, 2257 (1993). 